\title{
Multilevel Coding in $M$-ary DPSK/Differential QAM High-Speed Optical Transmission With Direct Detection
}

\author{
Ivan B. Djordjevic, Member, IEEE, and Bane Vasic, Senior Member, IEEE
}

\begin{abstract}
A bandwidth- and power-efficient modulation scheme using $M$-ary differential phase-shift keying (DPSK)/ differential quadrature amplitude modulation (DQAM) and low-density parity-check (LDPC) codes as component codes in a multilevel coding (MLC) is proposed for optical transmission systems with direct detection. An MLC scheme with $2 \mathrm{~b} / \mathrm{s} / \mathrm{Hz}$ spectral efficiency based on block-circulant component codes provides the coding gain of $12.3 \mathrm{~dB}$ when compared to uncoded 8-DPSK, and $8.3 \mathrm{~dB}$ when compared to uncoded 4-DPSK (QDPSK).
\end{abstract}

Index Terms-Differential quadrature amplitude modulation (DQAM), low-density parity-check codes (LDPC), $M$-ary differential phase-shift keying (DPSK), Mach-Zehnder interferometer, multilevel coding, optical communications.

\section{INTRODUCTION}

$\mathbf{M}$ ULTILEVEL coding (MLC), proposed by Imai and Hirakawa in 1977 [1], is a powerful coded modulation scheme capable of achieving both bandwidth- and powerefficient communication [2], [3]. The key idea behind the MLC is to protect the individual bits using different binary codes and use $M$-ary signal constellation (for more details, the reader is referred to [1]-[9]). The decoding is based on so-called multistage decoding (MSD) algorithm, in which decisions from prior (lower) decoding stages are passed to the next (higher) stages (see [4]). Despite its attractiveness with respect to large coding gain, the MLC with the MSD algorithm has serious limitation for use in high-speed applications, such as optical communications, which is due to the inherently large delay of the MSD algorithm. One possible solution is to use the parallel independent decoding (PID) [3]. In the MLC/PID scheme, the information bit stream is split into $L$ different levels, and the corresponding bits at different levels are encoded using different encoders, and then combined into a signal point using appropriate mapping rule. At the receiver side, decoders at different levels operate independently and in parallel. For more details on MLC in

Manuscript received May 4, 2005; revised September 5, 2005. This work is supported by the National Science Foundation under Grants ITR 0325979 and CCR 0208597.

I. B. Djordjevic is with the Department of Electrical and Computer Engineering, University of Arizona, Tucson, AZ 85721 USA, on leave from the Faculty of Computing, Engineering and Mathematical Sciences, University of the West of England, Bristol BS16 1QY, U.K. (e-mail: ivan@ece.arizona.edu).

B. Vasic is with the Department of Electrical and Computer Engineering, University of Arizona, Tucson, AZ 85721 USA (e-mail: vasic@ece. arizona.edu).

Digital Object Identifier 10.1109/JLT.2005.860148 general, an interesting reader is referred to [4], and for more details on PID to [3]. It has been widely recognized that the use of Gray mapping and PID at each level separately, with optimally chosen component codes [3], [5], [6], may lead to channel capacity approaching performance. Our idea is to use low-density parity-check (LDPC) codes as component codes. LDPC codes have been shown to be able to achieve impressive coding gains on a variety of channels, including the fiber-optic communication channel [10], [11]. Furthermore, their low-complexity decoding algorithm makes them a good choice for combining with the low-delay PID scheme.

The second ingredient of our scheme is a proper modulation format. Phase-shift keying (PSK) has attracted significant attention in late 1980s and early 1990s; however, due to stringent requirements for laser linewidths, precise alignment of transmitter and receiver center frequencies, and polarization sensitivity [12], [13], the interest for PSK optical transmission decreased rapidly, especially after the advent and deployment of erbium-doped fiber amplifiers (EDFAs). Recently, the direct detection of differential PSK (DPSK) with a Mach-Zehnder delay interferometer (MZDI) has generated significant research attention [14]-[16]. In addition, it has been shown [14] that return-to-zero (RZ)-DSPK can further facilitate the implementation and improve the nonlinearity tolerance of PSK. However, it is well known that [17], for a given constellation size (and $M>4$ ), quadrature amplitude modulation (QAM) is more power efficient at moderate to high signal-to-noise ratios (SNRs) on an additive white Gaussian noise (AWGN) channel. The superior performance of $M$-ary QAM can only be realized if the system operates in the regime of weak fiber nonlinearities. Bandwidth-efficient schemes considered so far in literature are designed for coherent PSK [1]-[9] and are not directly applicable in high-speed optical transmission with direct detection. The key technological issue to be solved before the practical implementation is to provide a method of a reasonable complexity that will provide sufficiently accurate amplitude estimates for QAM demodulation. Warrier and Madhow [18] and Chen et al. [19] give an excellent approach to a design of power-efficient amplitude/phase constellations and nearoptimal low-complexity implementation of noncoherent block demodulation. However, the complexity of a joint noncoherent demodulation and decoding scheme given in [19] is too high, and the code rate is too low for high-speed implementation.

In this paper, we propose transmitter and receiver configurations suitable for $M$-ary DPSK (M-DPSK) and $M$-ary 


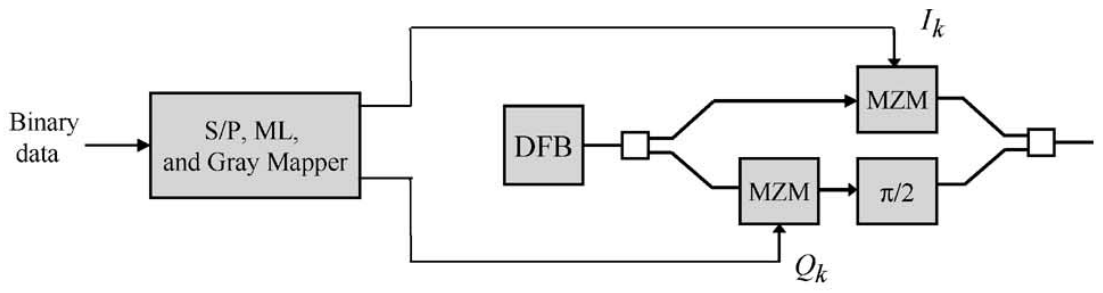

(a)

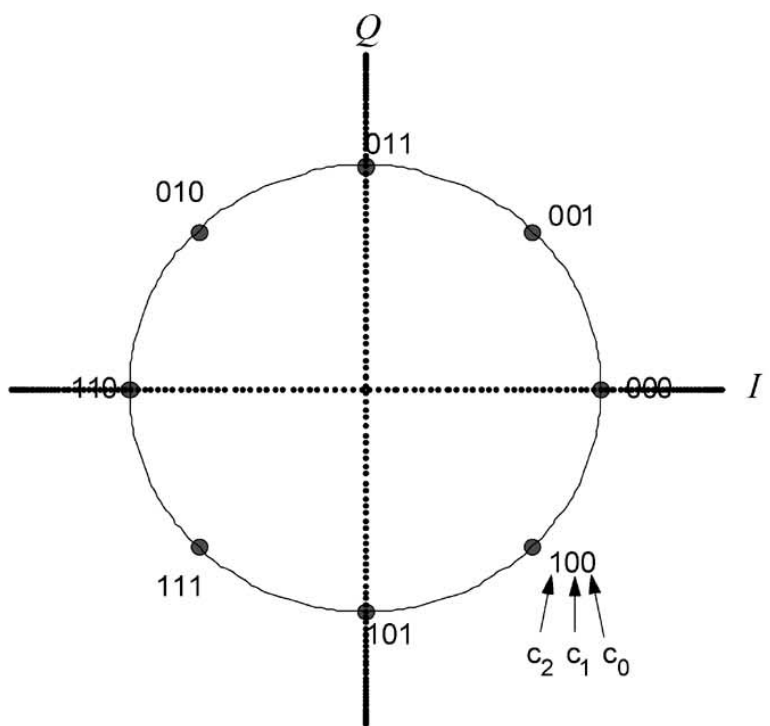

(b)

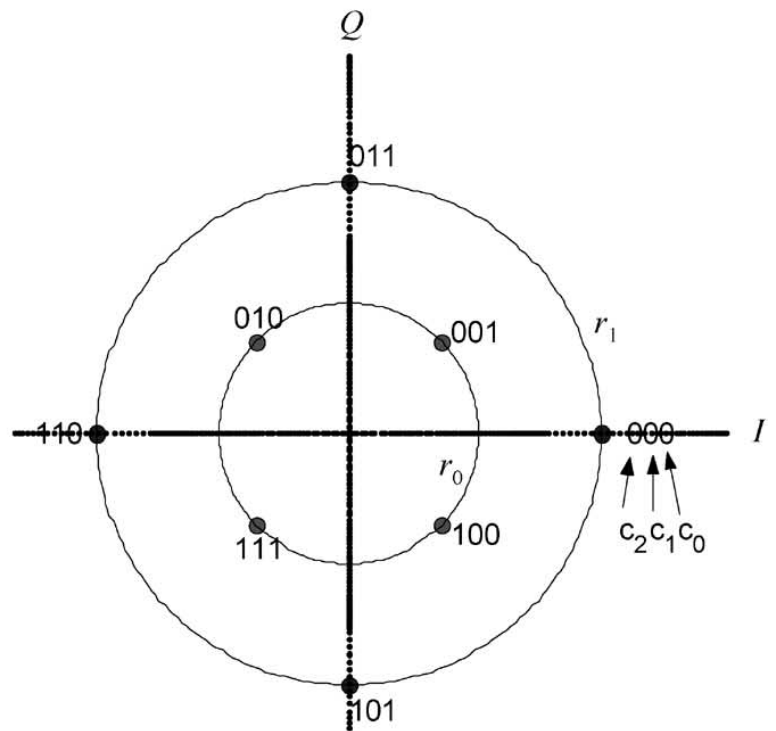

(c)

Fig. 1. (a) Optical M-DPSK/M-DQAM transmitter configuration. DFB—distributed feedback laser. MZM-Mach-Zehnder modulator. S/P—serial-to-parallel converter. ML—multilevel encoding. Gray mapper: M-DPSK or M-DQAM Gray mapping. (b) 8-DPSK constellation diagram. (c) 8-DQAM constellation diagram.

differential QAM (M-DQAM) optical transmission, and study LDPC codes suitable for MLC in bandwidth-efficient optical transmission with direct detection. Our transmitter and receiver configurations are based on the recent optical 4-DPSK (QDPSK) scheme [15], [16]. Two classes of LDPC codes, namely, random like and structured, both with spectral efficiency of $2 \mathrm{~b} / \mathrm{s} / \mathrm{Hz}$ or higher, are investigated.

\section{M-DPSK/M-DQAM TRANSMITTER/RECEIVER CONFIGURATION}

Optical M-DPSK and M-DQAM modulations achieve the transmission of $\log _{2} M(=L)$ bits per symbol, providing power- and bandwidth-efficient communication. The block scheme of a transmitter is shown in Fig. 1, which is, in fact, the generalized QDPSK transmitter scheme proposed in [15]. Since the modulation is differential, at each $k$ th transmission interval, the data phasor $\phi_{k}=\phi_{k-1}+\Delta \phi_{k}$ is sent, where $\Delta \phi_{k} \in\{0,2 \pi / M, \ldots, 2 \pi(M-1) / M\}$ is determined by the sequence of $\log _{2} M$ input bits using the Gray mapping rule [17]. As an illustration, the 8-DPSK and 8-DQAM constellation diagrams are given in Fig. 1(b) and (c), respectively, and the corresponding Gray mapping rule is given in Table I. The transmitted signal may be written in complex notation as

$$
s(t)=\sum_{k} I_{k} g(t-k T)+j Q_{k} g(t-k T)
$$

TABLE I

8-DSPK/8-DQAM GRAY MAPPING RULE

\begin{tabular}{|c||c||c|c||c|c||}
\hline \multirow{2}{*}{ Input $\left(c_{2} c_{1} c_{0}\right)$} & \multirow{2}{*}{$\Delta \phi_{k}$} & \multicolumn{2}{c||}{ 8-DPSK } & \multicolumn{2}{c||}{ 8-DQAM } \\
\cline { 3 - 6 } & & $I_{k}$ & $Q_{k}$ & $I_{k}$ & $Q_{k}$ \\
\hline \hline 000 & 0 & 1 & 0 & $1+\sqrt{ } 3$ & 0 \\
\hline 001 & $\pi / 4$ & $\sqrt{ } 2 / 2$ & $\sqrt{ } 2 / 2$ & 1 & 1 \\
\hline 011 & $\pi / 2$ & 0 & 1 & 0 & $1+\sqrt{ } 3$ \\
\hline 010 & $3 \pi / 4$ & $-\sqrt{ } 2 / 2$ & $\sqrt{ } 2 / 2$ & -1 & 1 \\
\hline 110 & $\pi$ & -1 & 0 & $-(1+\sqrt{ } 3)$ & 0 \\
\hline 111 & $5 \pi / 4$ & $-\sqrt{ } 2 / 2$ & $-\sqrt{ } 2 / 2$ & -1 & -1 \\
\hline 101 & $3 \pi / 2$ & 0 & -1 & 0 & $-(1+\sqrt{ } 3)$ \\
\hline 100 & $7 \pi / 4$ & $\sqrt{ } 2 / 2$ & $-\sqrt{ } 2 / 2$ & 1 & -1 \\
\hline
\end{tabular}

where $I_{k}$ and $Q_{k}$ are given in Table I $\left(j^{2}=-1, g(t)\right.$ is the pulse shape). The average transmitted power for 8 -DPSK is $A^{2}$, and that for 8 -DQAM is $4.73 A^{2}$. The coordinates of signal points are normalized by $A$. In the simulations, $A$ is chosen in such a way that both 8-DPSK and 8-DQAM have the same average power.

As we recently pointed out [20], any amplitude/phase constellation can be obtained by employing a single dual-drive (push-pull) Mach-Zehnder modulator (MZM) instead of a conventional transmitter with two MZMs [Fig. 1(a)].

The M-DPSK and M-DQAM receiver configuration is given in Fig. 2, which is, again, the generalization of QDPSK receiver configuration from [15] and [16]. It is straightforward to show that for the receiver input $E_{k}=\left|E_{k}\right| \exp \left(\mathrm{j} \varphi_{k}\right)$, the outputs of upper and lower branches are proportional to $\operatorname{Re}\left\{E_{k} E_{k-1}^{*}\right\}$ and 


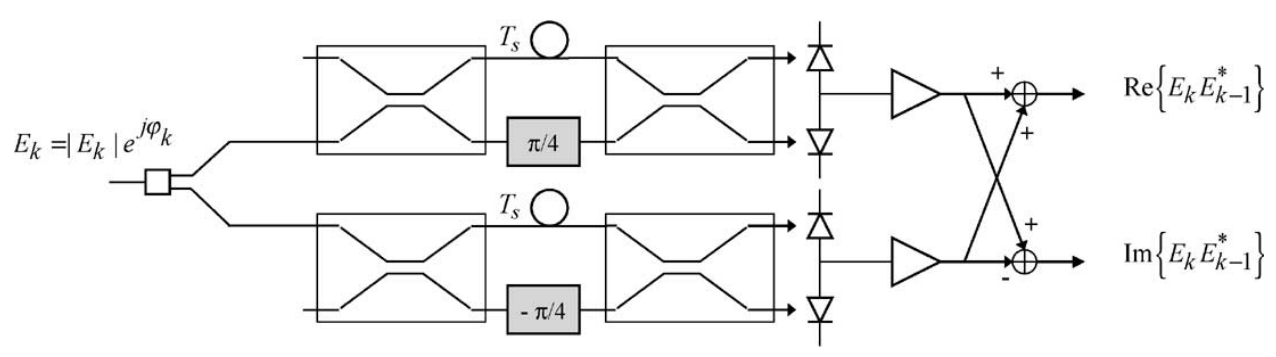

Fig. 2. M-DPSK/M-DQAM receiver configuration based on MZDIs. ( $T_{s}$ denotes the symbol duration.)

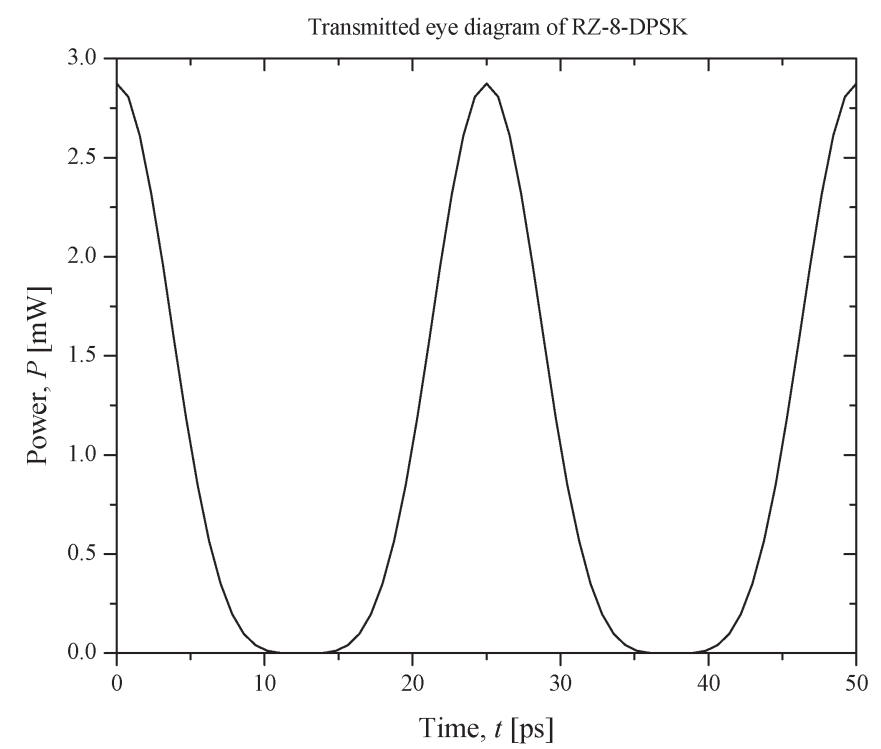

(a)

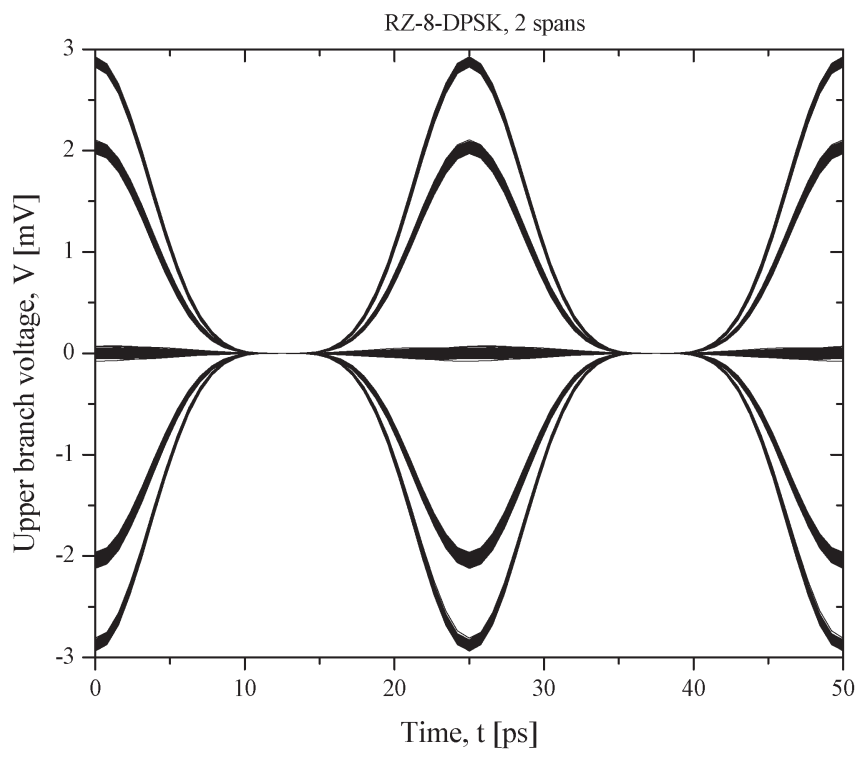

(b)

Fig. 3. (a) Transmitted and (b) received (upper branch of Fig. 2) eye diagrams of RZ-8-DPSK at 40 Gsymbols/s.

$\operatorname{Im}\left\{E_{k} E_{k-1}^{*}\right\}$, respectively. In principle, the phase difference $\Delta \varphi_{k}=\varphi_{k}-\varphi_{k-1}$ can be determined from the ratio of the lower and upper branch outputs by

$$
\frac{\operatorname{Im}\left\{E_{k} E_{k-1}^{*}\right\}}{\operatorname{Re}\left\{E_{k} E_{k-1}^{*}\right\}}=\frac{\left|E_{k}\right|\left|E_{k-1}\right| \sin \Delta \varphi_{k}}{\left|E_{k}\right|\left|E_{k-1}\right| \cos \Delta \varphi_{k}}=\tan \Delta \varphi_{k}
$$

Notice that the phase difference can be determined from the lower and upper branch signals even when the amplitude of pulses into two consecutive intervals $\left|E_{k}\right|$ and $\left|E_{k-1}\right|$ are different. Therefore, the arbitrary QAM constellation, including square constellation, can be applied, provided that no pair of signal points has the same phase. The above approach is given for the sake of explanation, but it is not used in simulations because of problems with uniqueness of $\tan \Delta \varphi_{k}$.

The Euclidean distance between neighboring points of the star 8-DQAM constellation in Fig. 1(c) is 2, provided that the radius of the inner circle is $\sqrt{ } 2$, and the radius of the outer circle is $1+\sqrt{ } 3$. It is known to be the best 8-QAM constellation, since it requires the least power for a given minimum distance among neighboring constellation points [17]. Corresponding Euclidean distance of 8-DPSK constellation in Fig. 1(b) is $2 A \sin (\pi / 8)$ (to keep the same average power, $A$ is chosen to be $\sqrt{ } 4.73$ ), and, therefore, an 8-DQAM system operating at $10 \mathrm{Gsymbols/ \textrm {s }}$ is expected to perform better than an 8-DPSK one in the regime of weak nonlinearities. At 40 Gsymbols/s, the 8-DQAM-based system is more sensitive to self-phase modulation (SPM) and intrachannel four-wave mixing (FWM), because different signal points are transmitted with different peak powers. However, to fully exploit the advantages of DQAM, a modified block differential encoding is to be used. This block encoding is proposed in [18] and accounts for the noncoherent metric. Instead of being used to determine the transmitted phase according to (2), the receiver outputs (upper and lower branches of Fig. 2) are now processed by the soft parallel independent decoders as shown in Fig. 6(b). Notice that proposed transmitter and receiver configurations are valid for any $M$ and any soft decision decoding, while the recently proposed 8-DPSK scheme [21] is suitable only for implementation with hard decision forward error correction (FEC) schemes.

As an illustration of the validity of proposed transmitter and receiver configurations, eye diagrams of a transmitted and a received RZ-8-DPSK signal at 40 Gsymbols/s (after two spans of dispersion map given in Section IV and launched power of $0 \mathrm{dBm}$ ) are shown in Fig. 3. The average power spectral densities of RZ-DPSK and RZ-8-DPSK are shown in Fig. 4. The eye diagrams of a transmitted and a received 8-DQAM signals are shown in Fig. 5(a), (b), while the power spectral density is given in Fig. 5(c). Notice that the main lobe widths of 8-DPSK and 8-DQAM spectra are three times lower than that of DPSK. 


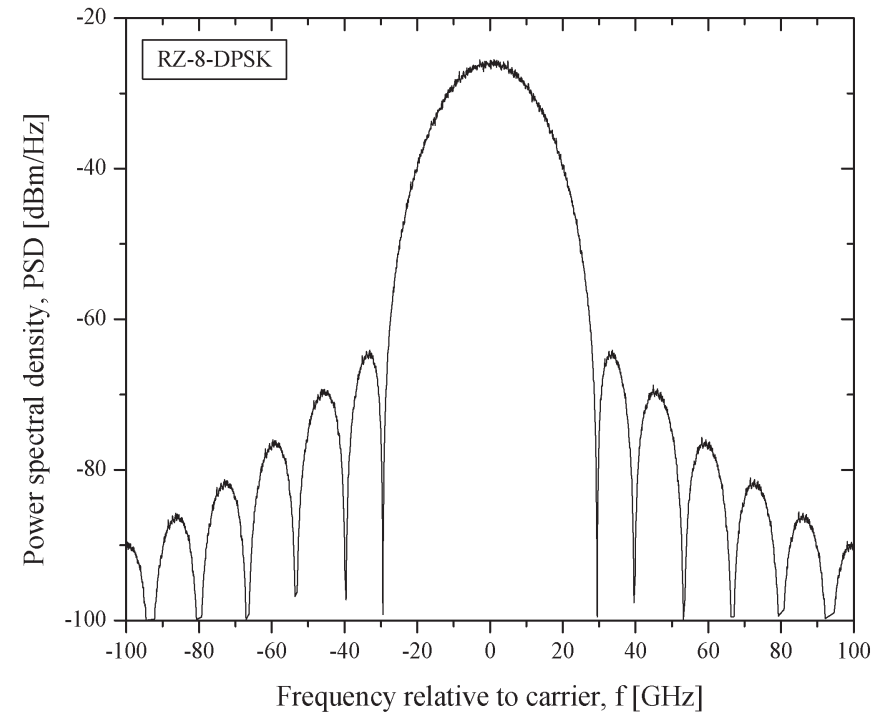

(a)

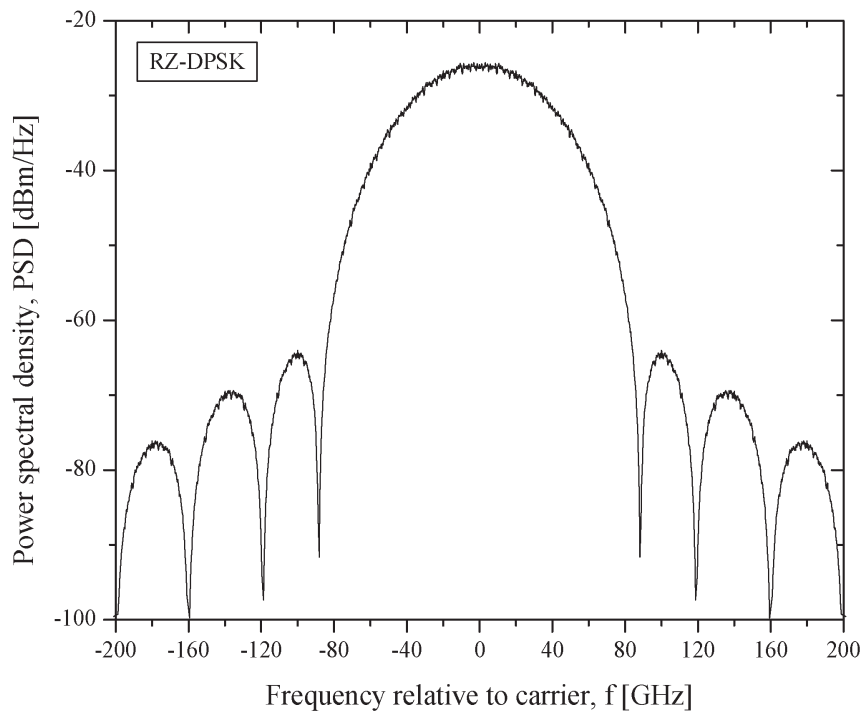

(b)

Fig. 4. Power spectral densities of (a) RZ-8-DPSK and (b) RZ-DPSK at 40 Gsymbols/s (duty cycle: 0.33).

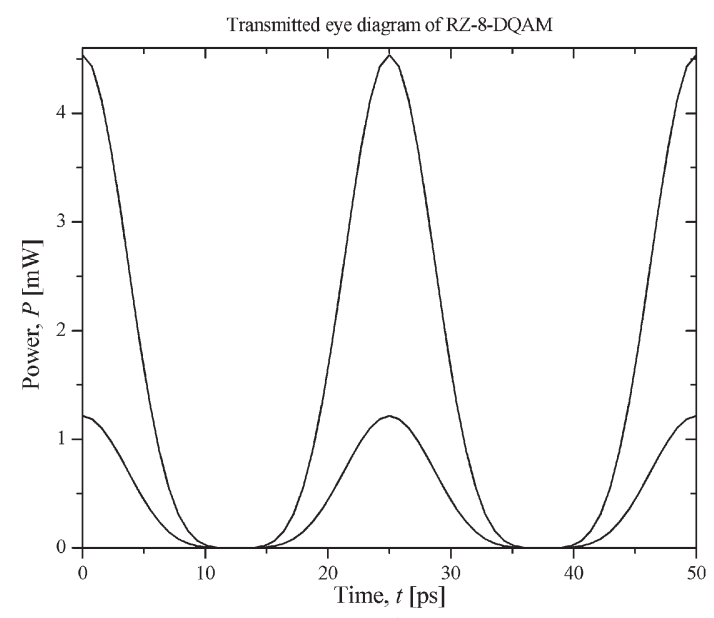

(a)

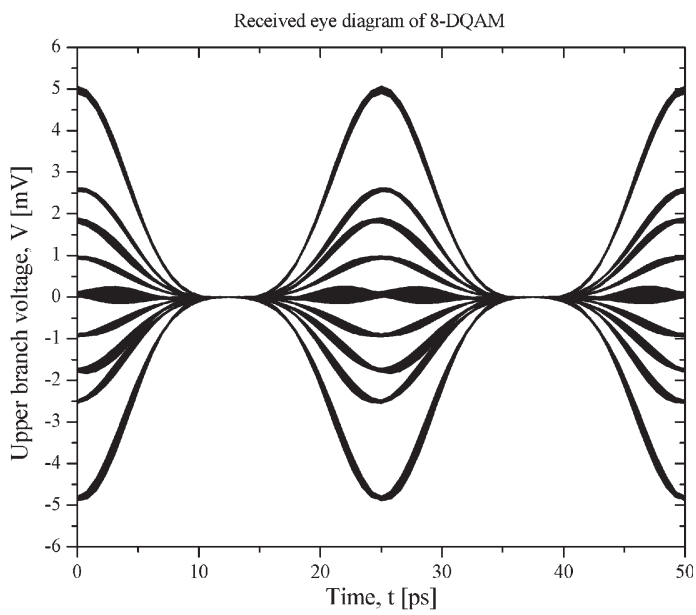

(b)

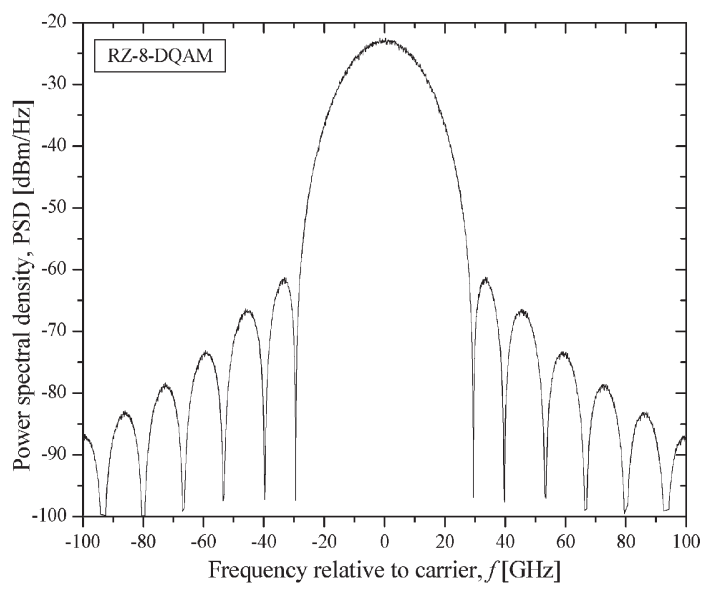

(c)

Fig. 5. (a) Transmitted and (b) received (upper branch of Fig. 2) eye diagrams of 8-DQAM at 40 Gsymbols/s. (c) Power spectral density of RZ-8-DQAM.

\section{Multilevel Coding}

The block diagram of the proposed MLC/PID scheme is shown in Fig. 6. The source bit stream is split into $L\left(=\log _{2} M\right)$ different levels, and the corresponding bits at different levels $u_{i}(i=0,1, \ldots, L-1)$ are encoded using different LDPC encoders, and then combined into a signal point using the Gray mapping rule and differential encoding as explained in Section II (see Table I). Two orthogonal 


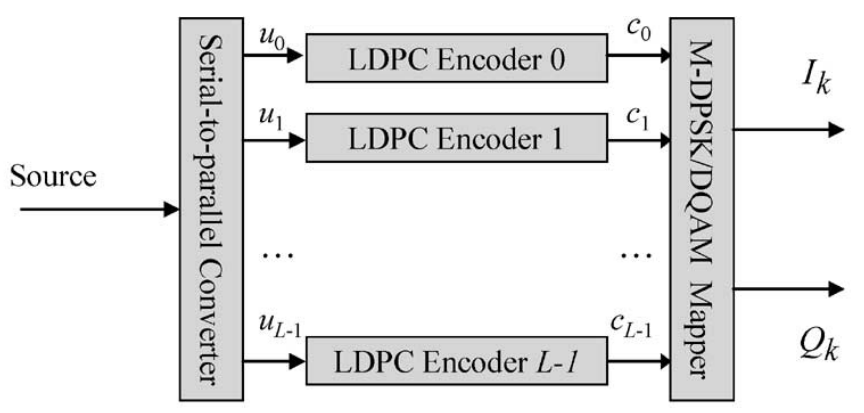

(a)

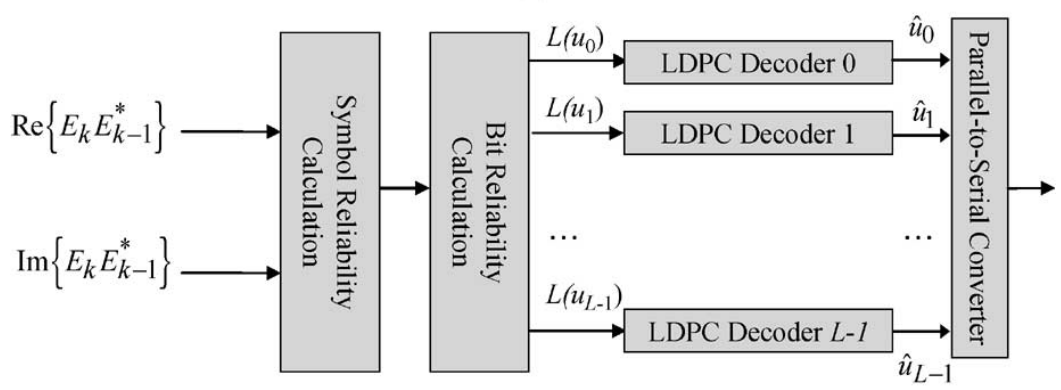

(b)

Fig. 6. Block diagram of MLC/PID. (a) Multilevel encoder. (b) Parallel independent decoders.

electrical streams (in-phase $I_{k}$ and quadrature $Q_{k}$ ) are driven to the RF inputs of MZMs according to Fig. 1(a). Each bit $c_{i}, i=0,1, \ldots, L-1$, is protected by a different LDPC code of rate $R_{i}=k_{i} / n$, where $k_{i}$ is the information word length of the $i$ th component code and $n$ is the codeword length. The Gray mapping device maps a binary vector $\left(c_{0}, c_{1}, \ldots\right.$, $\left.c_{L-1}\right)$ into a signal point $\left(I_{k}, Q_{k}\right)$. As an illustration, the Gray mapping rules for 8-DPSK and for 8-DQAM constellations are given in Fig. 1(b) and (c) and Table I.

It is straightforward to show [3] that the spectral efficiency $R_{s}$ is equal to the sum of the individual code rates $R_{i}=k_{i} / n$

$$
R_{s}=\sum_{i=0}^{L-1} R_{i}=\frac{1}{n} \sum_{i=0}^{L-1} k_{i}=\frac{k}{n}, \quad k=\sum_{i=0}^{L-1} k_{i} .
$$

In a receiver, all LDPC decoders operate independently and in parallel. The LDPC decoders input reliabilities $L\left(u_{j}\right)$, which are calculated from the symbol reliabilities $\lambda(\boldsymbol{u}), \boldsymbol{u}=$ $\left(u_{0}, u_{1}, \ldots, u_{L-1}\right)$ as

$$
L\left(u_{j}\right)=\log \frac{\sum_{\boldsymbol{u}: u_{j}}=1^{\exp [\lambda(\boldsymbol{u})]}}{\sum_{\boldsymbol{u}: u_{j}}=0^{\exp [\lambda(\boldsymbol{u})]}}
$$

while the symbol reliabilities are calculated from channel samples $\boldsymbol{r}=(\boldsymbol{x}, \boldsymbol{y})$ ( $\boldsymbol{x}$ and $\boldsymbol{y}$ are obtained by sampling the outputs of upper and lower branches in Fig. 2, respectively)

$$
\lambda(\boldsymbol{u})=\log \frac{P(\boldsymbol{u} \mid \boldsymbol{r})}{P(0 \mid \boldsymbol{r})} .
$$

TABLE II

MLC/PID SCHEMES EMPLOYED IN SIMULATIONS

\begin{tabular}{||c||c||c||c||c||}
\hline $\begin{array}{c}\text { MLC/PID } \\
\text { based on }\end{array}$ & Level 0 & Level 1 & Level 2 & $\begin{array}{c}\text { Spectral } \\
\text { efficiency } \\
\text { bits/s/Hz] }\end{array}$ \\
\hline \hline $\begin{array}{c}\text { MacKay } \\
\text { codes }\end{array}$ & $(1908,1697)$ & $(1908,1697)$ & $(1908,1697)$ & 2.668 \\
\hline $\begin{array}{c}\text { MacNeish- } \\
\text { Mann codes }\end{array}$ & $(1225,1088)$ & $(1225,1088)$ & $(1225,1088)$ & 2.664 \\
\hline Array codes & $(1369,703)$ & $(1369,1036)$ & $(1369,1036)$ & 2.027 \\
\hline $\begin{array}{c}\text { Block- } \\
\text { circulant 1 }\end{array}$ & $(4320,3242)$ & $(4320,3242)$ & $(4320,3242)$ & 2.251 \\
\hline $\begin{array}{c}\text { Block- } \\
\text { circulant 2 }\end{array}$ & $(4320,2160)$ & $(4320,3242)$ & $(4320,3242)$ & 2 \\
\hline
\end{tabular}

In (4), the summation is done over all vectors in which the $j$ th component is 1 for the numerator and 0 for the denominator. $P(\boldsymbol{u} \mid \boldsymbol{r})$ is determined using Bayes' rule

$$
P(\boldsymbol{u} \mid \boldsymbol{r})=\frac{P(\boldsymbol{r} \mid \boldsymbol{u}) P(\boldsymbol{u})}{\sum_{\boldsymbol{v}} P(\boldsymbol{r} \mid \boldsymbol{v}) P(\boldsymbol{v})}
$$

and $P(\boldsymbol{r} \mid \boldsymbol{u})$ is estimated from the channel by determination of histograms. In the calculation of LDPC decoder input reliabilities $L\left(u_{j}\right)$, the "max-star" operator is applied recursively [22] as

$$
\max ^{*}(x, y)=\max (x, y)+\log \left(1+e^{-|x-y|}\right)
$$

where the $\max ^{*}$ is defined as $\max ^{*}(x, y)=\log \left(e^{x}+e^{y}\right)$.

\section{NumERicAl RESUlts}

The simulation results for different LDPC component codes, described in Table II, for 8-DPSK scheme with Gray mapping 


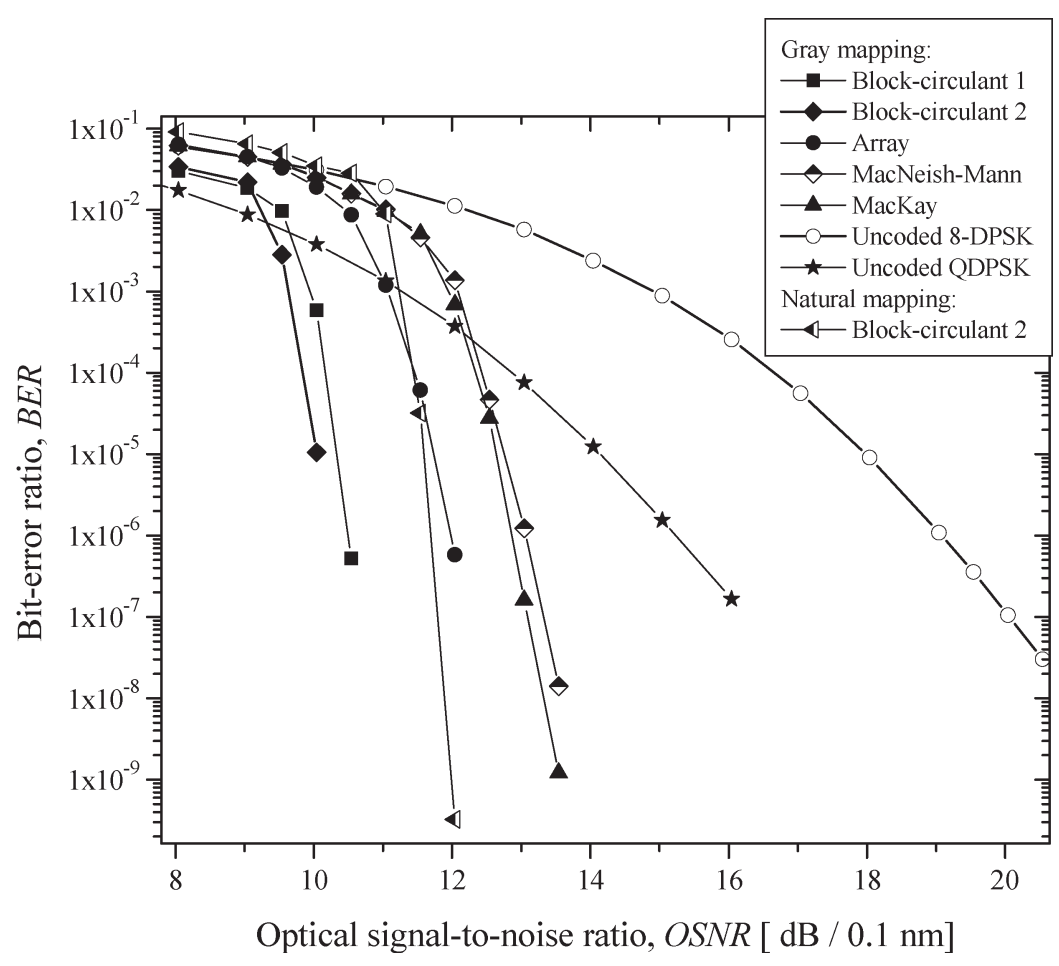

Fig. 7. BER performance of MLC/PID with 8-DPSK for AWGN channel model at 40 Gsymbols/s based on the Gray mapping rule.

at 40 Gsymbols/s are shown in Fig. 7. The uncoded curves are obtained from (4) by

$$
\hat{u}_{j}= \begin{cases}1, & \text { if } L\left(u_{j}\right)>0 \\ 0, & \text { otherwise }\end{cases}
$$

Since in fiber-optic communications, the optical SNR (OSNR) is more commonly used as a figure of merit than the SNR, SNR per bit is translated into OSNR with $0.1 \mathrm{~nm}$ resolution bandwidth when both polarizations are taken into account, similarly as in [14]. Two types of LDPC codes, namely, random-like codes [23] and structured codes [24]-[26], are considered as component codes for several spectral efficiencies. Interestingly, for the same spectral efficiency, the scheme based on a longer random code [23] performs the same as shorter structured code [24]. The MLC/PID scheme of spectral efficiency $2.251 \mathrm{~b} / \mathrm{s} / \mathrm{Hz}$ based on block-circulant component codes [25] provides the coding gain of $9.45 \mathrm{~dB}$ at a bit error rate (BER) of $10^{-7}$. The expected coding gain at a BER of $10^{-10}$ is $\sim 12.1 \mathrm{~dB}$. The MLC/PID scheme with blockcirculant codes [25] significantly outperforms the one based on array codes [26]. At a BER of $10^{-10}$, the expected coding gain of the block-circulant MLC/PID scheme of spectral efficiency $2 \mathrm{~b} / \mathrm{s} / \mathrm{Hz}$ is around $12.3 \mathrm{~dB}$ when compared to uncoded 8 -DPSK, and $\sim 8.3 \mathrm{~dB}$ when compared to uncoded QDPSK. Gray mapping demonstrates superiority over natural mapping (with an improvement of approximately $1.6 \mathrm{~dB}$ at a BER of $10^{-10}$, and larger improvements for lower BER values).

MLC/PID BER performance for different signal mappings is shown in Fig. 8. For the sake of conciseness (similarly as in [27]), we use the eight-component vector to represent an 8-DPSK mapper, where every component is a decimal repre- sentation of corresponding binary bits mapped in that signal point. For example, the Gray mapping in Fig. 1(b) (or Table I) may be represented as $(0,1,3,2,6,7,5,4)$. The maximum squared Euclidean weight (MSEW) mapping doped with $50 \%$ of Gray mapping (proposed in [27]) performs better than natural mapping, but still worse than Gray mapping.

For the 8-DPSK scheme with spectral efficiency of $2 \mathrm{~b} / \mathrm{s} / \mathrm{Hz}$, the optimum distribution of component code rates is used as explained in [3]. However, to keep the hardware complexity low, we use highly structured LDPC codes as opposed to irregular codes with optimum node degree distribution that are used in [3]. Notice that the performance can be further improved by the following methods: 1) optimizing the code rate ratio for 8-DPSK; 2) using optimum irregular codes; 3 ) using the optimum mapping rule; and 4) using the optimum dispersion map. Of course, this gain is possible at the expense of increased complexity. Unfortunately, highly irregular LDPC codes are difficult to implement at bit rates of interest in fiberoptic communications (10 or $40 \mathrm{~Gb} / \mathrm{s}$ ).

The results of time-extensive Monte Carlo simulations for the dispersion map in Fig. 9 are shown in Fig. 10. The dispersion map is composed of $N$ spans of length $L=48 \mathrm{~km}$, each span consisting of $2 L / 3 \mathrm{~km}$ of $\mathrm{D}_{+}$fiber followed by $L / 3 \mathrm{~km}$ of $\mathrm{D}_{\text {- fiber. }} \mathrm{D}_{+}$fiber has a dispersion of $20 \mathrm{ps} /(\mathrm{nm} \cdot \mathrm{km})$, a dispersion slope of $0.06 \mathrm{ps} /\left(\mathrm{nm}^{2} \cdot \mathrm{km}\right)$, an effective area equal to $110 \mu \mathrm{m}^{2}$, and loss equal to $0.19 \mathrm{~dB} / \mathrm{km}$. The $\mathrm{D}_{-}$fiber has a dispersion of $-40 \mathrm{ps} /(\mathrm{nm} \cdot \mathrm{km})$, a dispersion slope of $-0.12 \mathrm{ps} /\left(\mathrm{nm}^{2} \cdot \mathrm{km}\right)$, an effective area equal to $30 \mu \mathrm{m}^{2}$, and loss equal to $0.25 \mathrm{~dB} / \mathrm{km}$. The nonlinear Kerr coefficient is set to $2.6 \times 10^{-20} \mathrm{~m}^{2} / \mathrm{W}$ in both types of fibers. The precompensation of $-320 \mathrm{ps} / \mathrm{nm}$ and corresponding postcompensation of $320 \mathrm{ps} / \mathrm{nm}$ are also employed. The EDFAs with a noise figure 


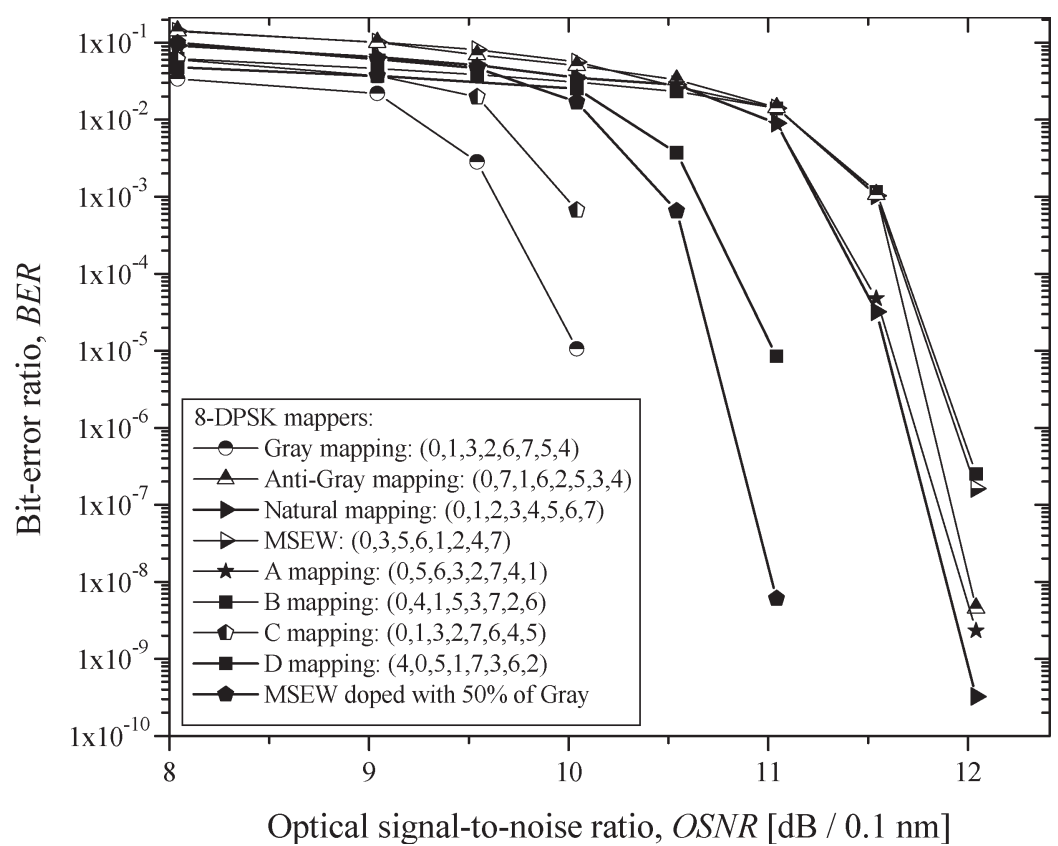

Fig. 8. MLC/PID BER performance for different symbol mappings at 40 Gsymbols/s.

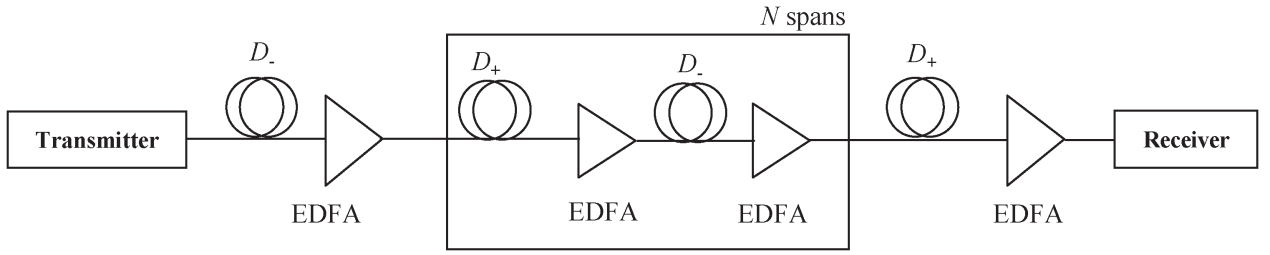

Fig. 9. Dispersion map under study.

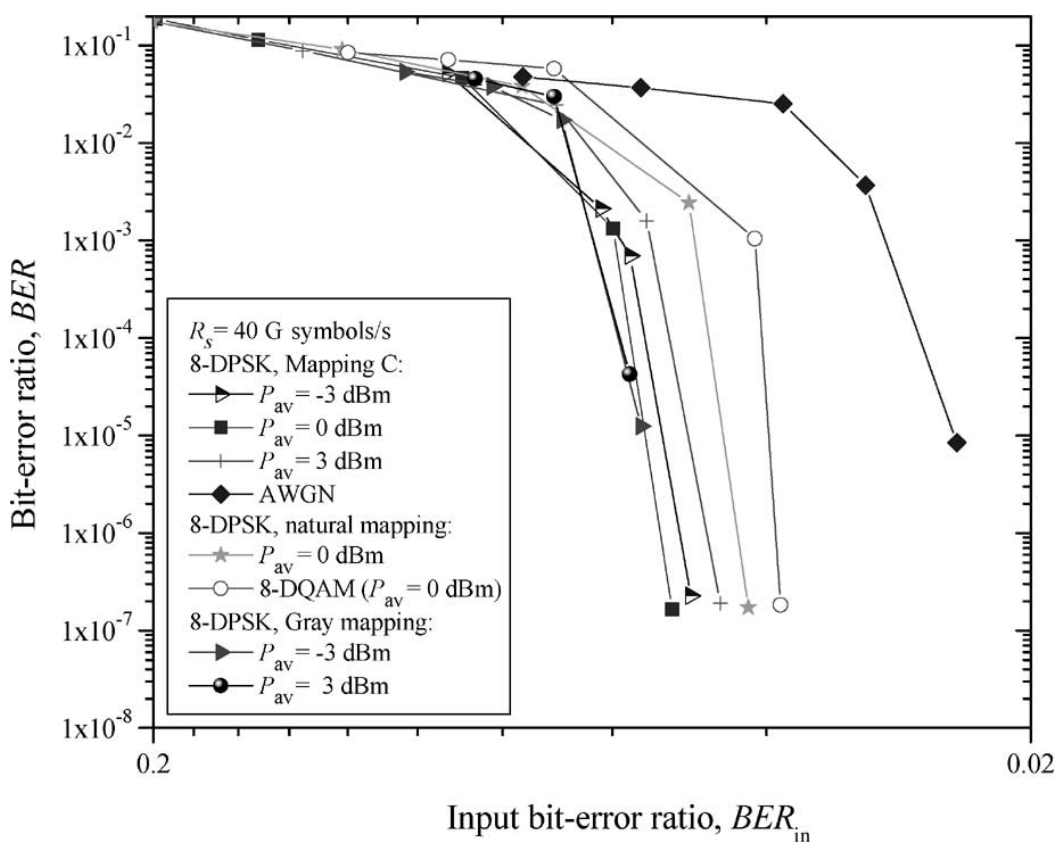

Fig. 10. BER performance of 2-b/s/Hz block-circulant MLC/PID with 8-DPSK and 8-DQAM for the dispersion map in Fig. 9 at 40 Gsymbols/s.

of $5 \mathrm{~dB}$ are deployed after every fiber section. The simulations were carried out with the launched powers per symbol $-3,0$, and $3 \mathrm{dBm}$, and a central wavelength of $1552.524 \mathrm{~nm}$. 8-DPSK and 8-DQAM with RZ pulses of duty cycle $33 \%$ are con- sidered. The propagation of a signal through the transmission media is modeled by a nonlinear Schrödinger equation [28] solved using the split-step Fourier method. The effects of Kerr nonlinearities (SPM, intrachannel cross-phase modulation, and 
intrachannel FWM), stimulated Raman scattering, dispersion [group-velocity dispersion (GVD) and second-order GVD], crosstalk, and intersymbol interference are taken into account. Similarly, as in ON-OFF-OFF keying (OOK) optical transmission [11], AWGN channel model underestimates BER performance. In the presence of intrachannel nonlinearities, 8-DPSK performs better than 8-DQAM. Probability density functions determined from simulations are different for different launched powers, resulting in a slightly different BER performance in Fig. 10. For Gray mapping, the differences in BER performance are negligible. Notice that the MLC design is done independently of the design of the dispersion map, and it would be interesting to investigate if a joint optimization leads to further performance improvements; however, this problem is beyond the scope of the paper.

In calculation of BER performance, shown in Fig. 10, an encoded sequence of length $2^{15}$ is transmitted many times over the whole transmission system for different amplified spontaneous emission (ASE) noise realizations. The number of spans is changed from 20 to 100 depending on modulation format and launched power. In calculation of initial likelihoods, the probability density functions determined from the simulation are employed.

The results of the simulations are obtained by maintaining a double precision for soft information. In our recent paper [29], we have shown that proper choice in number of quantization bits results in a negligible BER performance loss.

The efficient realization of the sum-product decoding algorithm proposed in [30] is employed in the simulations. Decoding halts when a valid codeword is generated or 25 iterations has been reached.

\section{CONCLUSION}

In conclusion, the MLC scheme based on LDPC codes as component codes is proposed for use in optical M-DPSK and M-DQAM communication systems with direct detection. For spectral efficiency of $2 \mathrm{~b} / \mathrm{s} / \mathrm{Hz}$, the proposed 8-DPSK scheme based on block-circulant LDPC codes as component codes and the Gray mapping rule provides the coding gain of $12.3 \mathrm{~dB}$ (at a BER of $10^{-10}$ ) with respect to an uncoded 8-DPSK. Notice that it significantly outperforms $\left(\sim 6 \mathrm{~dB}\right.$ at $\left.10^{-13}\right)$ the recently proposed optical trellis-coded modulation scheme [31]. It should be also noted that the overhead of our codes is $33 \%$ or less, while the code in [31] has an overhead of $100 \%$. Based on the recently proposed QDPSK scheme [15], [16], the transmitter and receiver configurations are generalized for use in any M-DPSK and M-DQAM optical communication system with direct detection.

\section{ACKNOWLEDGMENT}

The authors thank the anonymous reviewers whose comments led to a significant improvement of the paper.

\section{REFERENCES}

[1] H. Imai and S. Hirakawa, "A new multilevel coding method using error correcting codes," IEEE Trans. Inf. Theory, vol. IT-23, no. 3, pp. 371377, May 1977.
[2] S. Benedetto, D. Divsalar, G. Montorsi, and H. Pollara, "Parallel concatenated trellis coded modulation," in Proc. IEEE Int. Conf. Communications, Dallas, TX, 1996, pp. 974-978.

[3] J. Hou, P. H. Siegel, L. B. Milstein, and H. D. Pfitser, "Capacityapproaching bandwidth-efficient coded modulation schemes based on low-density parity-check codes," IEEE Trans. Inf. Theory, vol. 49, no. 9, pp. 2141-2155, Sep. 2003.

[4] U. Wachsmann, R. F. H. Fischer, and J. B. Huber, "Multilevel codes: Theoretical concepts and practical design rules," IEEE Trans. Inf. Theory, vol. 45, no. 5, pp. 1361-1391, Jul. 1999.

[5] J. Hou and M. H. Lee, "Multilevel LDPC codes design for semi-BICM," IEEE Commun. Lett., vol. 8, no. 11, pp. 674-676, Nov. 2004.

[6] P. Limpaphayom and K. A. Winick, "Power- and bandwidth-efficient communications using LDPC codes," IEEE Trans. Commun., vol. 52, no. 3, pp. 350-354, Mar. 2004.

[7] K. R. Narayanan and J. Li, "Bandwidth efficient low density parity check codes using multilevel coding and iterative multistage decoding," in Proc. 2nd Symp. Turbo Codes and Related Topics, Brest, France, 2000, pp. $165-168$.

[8] N. Varnica, X. Ma, and A. Kavcic, "Iteratively decodable codes for bridging the shaping gap in communication channels," in Proc. Asilomar Conf. Signals, Systems, Computers, Pacific Grove, CA, Nov. 2002, pp. 3-7.

[9] Y. Yi and M. H. Lee, "Optimized low-density parity-check (LDPC) codes for bandwidth efficient modulation," in Proc. 2nd Int. Symp. Turbo Codes and Related Topics, Brest, France, Jul. 2000, pp. 165-168.

[10] T. J. Richardson, A. Shokrollahi, and R. Urbanke, "Design of capacityapproaching irregular low-density parity-check codes," IEEE Trans. Inf. Theory, vol. 47, no. 2, pp. 619-637, Feb. 2001.

[11] I. B. Djordjevic, S. Sankaranarayanan, and B. Vasic, "Projective plane iteratively decodable block codes for WDM high-speed long-haul transmission systems," J. Lightw. Technol., vol. 22, no. 3, pp. 695-702, Mar. 2004.

[12] G. Jacobsen, Noise in Digital Optical Transmission Systems. Boston, MA: Artech House, 1994.

[13] M. Cvijetic, Coherent and Nonlinear Lightwave Communications. Boston, MA: Artech House, 1996.

[14] C. Xu, X. Liu, and X. Wei, "Differential phase-shift keying for highspectral efficiency optical transmission," IEEE J. Sel. Topics Quantum Electron., vol. 10, no. 2, pp. 281-293, Mar./Apr. 2004.

[15] R. A. Griffin and A. C. Carter, "Optical differential quadrature phaseshift keying (oDQPSK) for high capacity optical transmission," in Proc. Optical Fiber Communication (OFC), Anaheim, CA, 2002, pp. 367-368.

[16] R. A. Griffin, R. G. Walker, and R. I. Johnstone, "Integrated devices for advanced modulation formats," in Proc. IEEE/LEOS Workshop Advanced Modulation Formats, San Francisco, CA, 2004, pp. 39-40.

[17] J. G. Proakis, Digital Communications. Boston, MA: McGraw-Hill, 2001.

[18] D. Warrier and U. Madhow, "Spectrally efficient noncoherent communication," IEEE Trans. Commun., vol. 48, no. 3, pp. 651-668, Mar. 2002.

[19] R.-R. Chen, R. Koetter, U. Madhow, and D. Agrawal, "Joint noncoherent demodulation and decoding for block fading channel: A practical framework for approaching Shannon capacity," IEEE Trans. Commun., vol. 51, no. 10, pp. 1676-1689, Oct. 2003.

[20] I. B. Djordjevic, J. Rorison, and S. Yu, "Chirped NRZ modulation format for high-speed transmission," J. Opt. Commun., vol. 25, no. 4, pp. 158160, Aug. 2004.

[21] M. Ohm, "Optical 8-DPSK and receiver with direct detection and multilevel electrical signals," in Proc. IEEE/LEOS Workshop Advanced Modulation Formats, San Francisco, CA, 2004, pp. 45-46.

[22] W. E. Ryan, "Concatenated convolutional codes and iterative decoding,' in Wiley Encyclopedia in Telecommunications, J. G. Proakis, Ed. New York: Wiley, 2003.

[23] D. J. C. MacKay, "Good error-correcting codes based on very sparse matrices," IEEE Trans. Inf. Theory, vol. 45, no. 2, pp. 399-431, Mar. 1999.

[24] I. B. Djordjevic and B. Vasic, "MacNeish-Mann theorem based iteratively decodable codes for optical communication systems," IEEE Commun. Lett., vol. 8, no. 8, pp. 538-540, Aug. 2004.

[25] O. Milenkovic, I. B. Djordjevic, and B. Vasic, "Block-circulant low-density parity-check codes for optical communication systems," IEEE/LEOS J. Sel. Topics Quantum Electron., vol. 10, no. 2, pp. 294-299, Mar./Apr. 2004.

[26] J. L. Fan, "Array codes as low-density parity-check codes," in Proc. 2nd Int. Symp. Turbo Codes and Related Topics, Brest, France, Sep. 2000, pp. 543-546.

[27] J. Tan and G. L. Stüber, "Analysis of symbol mappers for iteratively decoded BICM," IEEE Trans. Wireless Commun., vol. 4, no. 2, pp. 662 672, Mar. 2005. 
[28] G. P. Agrawal, Nonlinear Fiber Optics. San Diego, CA: Academic, 2001.

[29] S. Sankaranarayanan, I. B. Djordjevic, and B. Vasic, "Iteratively decodable codes on $m$-flats for WDM high-speed long-haul transmission," IEEE/OSA J. Lightw. Technol. submitted for publication.

[30] H. Xiao-Yu et al."Efficient implementations of the sum-product algorithm for decoding of LDPC codes," in Proc. IEEE Global Telecommunications (GLOBECOM), San Antonio, TX, Nov. 2001, vol. 2, pp. 1036-1036E.

[31] H. Buelow, G. Thielecke, and F. Buchali, "Optical trellis-coded modulation (oTCM)," presented at the Optical Fiber Communication (OFC), Los Angeles, CA, 2004, Paper WM5.

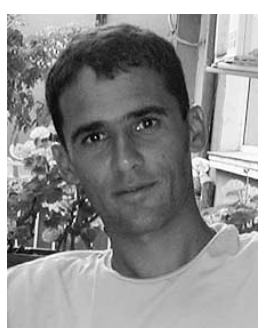

Ivan B. Djordjevic (M'04) received the Dipl.Ing., M.Sc., and Ph.D. degrees from the University of Nis, Nis, Serbia, all in electrical engineering, in 1994, 1997, and 1999, respectively.

From 1994 to 1996, he was with the University of Nis, and from 1996 to 2000, with the State Telecommunications Company (Serbia Telecom), District Office for Networks, Nis, Serbia. He was involved in digital transmission systems commissioning and acceptance, design, maintenance, installation, and connection. From 2000 to 2001, he was with the National Technical University of Athens, Athens, Greece, and with TyCom U.S. Inc. (now TyCo Telecommunications), Eatontown, NJ. He was involved in modeling and simulation of wavelength division multiplexing (WDM) systems and networks. During 2002 and 2003, he was with the University of Arizona, Tucson, University of Bristol, U.K., and University of the West of England, Bristol, U.K., working on forward error correction and iterative decoding for optical transmission, optical code division multiple access (CDMA), highspeed transmission, and optical switches. He is now with the University of Arizona, Tucson, on leave from the University of the West of England, Bristol, U.K. He is the author of more than 100 international journal articles and international conference papers. His research interests include dense WDM (DWDM) fiber-optic communication systems and networks, error control coding, orthogonal frequency division multiplexing (OFDM), free-space optics, CDMA, optical packet switching, coherent communications, statistical communication theory, and satellite and wireless communications.

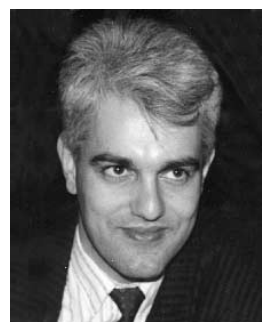

Bane Vasic (S'92-M'93-SM'02) received the B.Sc., M.Sc., and Ph.D. degrees from the University of Nis, Nis, Serbia, all in electrical engineering, in 1990, 1991, and 1994, respectively.

From 1996 to 1997, he worked as a Visiting Scientist at the Rochester Institute of Technology and Kodak Research, Rochester, NY, where he was involved in the research in optical storage channels. From 1998 to 2000, he was with Lucent Technologies, Bell Laboratories. He was involved in research coding schemes and architectures for high-speed applications. He was involved in the research in iterative decoding and lowdensity parity-check codes, as well as the development of codes and detectors implemented in Lucent (now Agere) chips. He is currently a Faculty Member of the Department of Electrical and Computer Engineering, University of Arizona, Tucson. His research interests include coding theory, information theory, communication theory, and digital communications and recording.

Dr. Vasic is a member of the Editorial Board of the IEEE TRANSACTIONS on MAgnetics. He served as the Technical Program Chair of the IEEE Communication Theory Workshop, 2003, and as a Co-Organizer of the Center for Discrete Mathematics and Theoretical Computer Science (DIMACS) Workshops on Optical/Magnetic Recording and Optical Transmission, and Theoretical Advances in Information Recording, 2004. He is a Co-Organizer of the Communication Theory Symposium within the International Conference on Communications (ICC 2006). 